\title{
Future directions for PET in colorectal cancer
}

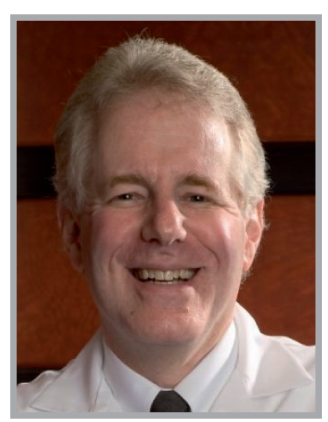

Anthony Frank Shields* talks to Ruth Williamson, Commissioning Editor: Dr Shields is Professor of Oncology and Medicine at Wayne University School of Medicine in Detroit (MI, USA). He is the Associate Center Director for Clinical Sciences and Program Leader in Molecular Imaging and Diagnostics at the Karmanos Cancer Institute. Dr Shields obtained his MD at Harvard Medical School (MA, USA) and PhD in cell biology at the Massachusetts Institute of Technology (MA, USA). He was trained in internal medicine and oncology at the University of Washington (WA, USA) and the Fred Hutchinson Cancer Research Center (WA, USA). He is involved in clinical research and the care of patients with colorectal and other gastrointestinal cancers. He is the sub-chair for colorectal cancer at the Southwest Oncology Group and serves on the National Cancer Institute Colon Task Force. His laboratory research focuses on the development and testing of tracers for use with PET for the assessment of a variety of tumors and their response to treatment. Dr Shields is helping to develop 3'-deoxy-3'-[18 $\mathrm{F}]$ fluorothymidine, a tracer for tumor proliferation, which is now being tested in centers worldwide, and works to incorporate innovative imaging in multicenter clinical trials.

Q How is PET currently used for the staging and evaluation of colorectal cancer (CRC)?

At present, the only tracer routinely used is fluorodeoxyglucose (FDG), which has been studied extensively for the last $10-15$ years and is routinely used in staging as an adjunct to standard computer tomography and MR. I would not say the use of PET-FDG for staging is a routine test we order on every patient every day, it is a test we use in addition to CT and MR when there are remaining questions.

We mainly use PET-FDG when there are possible lesions either visualized or suspected, in patients who are recently diagnosed when we are trying to determine whether those lesions are likely to be real or not, which is a very common problem. It is also a common problem in the re-evaluation and restaging of patients who have had previously diagnosed disease. We are frequently in the situation where patients have a limited number of metastasis to the liver or the lungs, who are being considered for resection with curative intent.

Initial trials, including the randomized trial done in The Netherlands a few years ago [1], looked at the utility of this approach and whether it avoided a necessary surgery. This trial found that PET was able to identify disease missed by the other techniques and could indicate whether or not the patient was a good surgical candidate. This trial also found that, in some cases, patients who were thought to have

*Departments of Oncology \& Medicine, Wayne State University, Karmanos Cancer Institute, MI, USA; shieldsa@karmanos.org

News \& Views

News

Journal Watch

Ask the Experts

Interviews "...for staging when there are
questions, PET is routine; for
restaging and patients with
possible metastatic disease
for resection, PET is certainly
routine. It saves the patients
the morbidity of unnecessary
surgery and it saves the
costs, which are substantial,
of unnecessary surgery."

Future Medicine ${ }_{\text {part of }}$ 


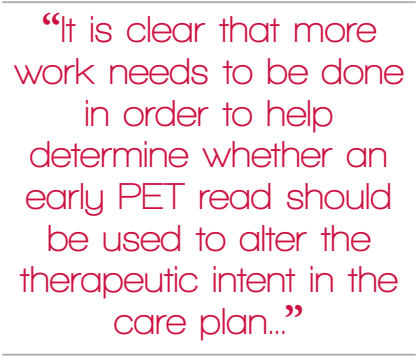

"It is clear that more determine whether an early PET read should be used to alter the care plan..." more extensive disease of lesions were actually not likely to have malignant disease. As such, I never take a patient to surgery for possible resections of recurrent disease or metastatic disease without doing a PET scan first, which is certainly one of the standard uses of PET.

Therefore, for staging when there are questions, PET is routine; for restaging and patients with possible metastatic disease for resection, PET is certainly routine. It saves the patients the morbidity of unnecessary surgery and it saves the costs, which are substantial, of unnecessary surgery.

\section{Q How is PET currently used for the treatment assessment of CRC?}

In terms of treatment assessment, PET has not yet become standard. We certainly use PET in some situations after patients have received chemotherapy for advanced disease. One can sometimes see the lesions have left activity but, at this time, we have not routinely incorporated that information into our routine assessment and how we evaluate and alter our therapy. This is still primarily done on the anatomic basis, in terms of whether there is growth of lesions or potential new lesions. It is clear that more work needs to be done in order to help determine whether an early PET read should be used to alter the therapeutic intent in the care plan; this is certainly an area in need of further evaluation.

Q You mentioned an important study in The Netherlands. Have there been any other recent studies of major significance to the field of PET for CRC?

The Netherlands study is certainly the most significant. Some studies looking at response evaluation, for instance in rectal cancer, have not dramatically changed how we treat such patients. Therefore, in general, we do not have any new studies that have really routinely affected the care we provide and the incorporation of PET using FDG.

Q Have there been any advances that may not yet be significant but show strong potential?

People have looked at other tracers. My laboratory helped develop 3'-deoxy-3'fluorothymidine (FLT) for imaging the
DNA synthetic pathway, which has been used in some rectal cancer studies [2]. They did not find in this situation that FLTPET was able to predict the pathologic response, but it may certainly be useful in understanding what the ultimate response and progression-free survival may be in the long term.

There are some suggestions in the literature that an FLT-PET response may demonstrate efficacy that is not reflected in early results, based on pathology or standard imaging techniques, which certainly requires further evaluation [3]. Therefore, people are certainly looking at other tracers, for use with PET and a number of venues, which include labeled drugs.

I think work with labeled drugs is one of the more interesting areas, with labeled PET tracers being developed alongside drugs to help determine which patients will benefit most from the agents. In some situations, studies are looking at whether or not a particular receptor is present; other studies are mainly looking at the delivery of the agent, and in some situations, studies are looking at whether the agent is taken up and retained within the tumor [4].

Therefore, this is an area of research that is being pursued for colorectal cancer (CRC), as well as other cancers, as people look to find markers that will help to determine whether their drugs are best used for one patient or another. We are interestingly dividing up diseases by both molecular approaches and imaging approaches to try to determine whether a drug is really going to benefit a patient. I think that some of the PET techniques will find their use in CRC and other cancers, to help sort out and predict when we should be giving a particular agent to the patient, which is one of the more exciting and difficult areas of research.

\section{Q You mentioned the development of new tracers - what are the most important things to consider when conducting such research?}

Developing a tracer is always fraught with a number of technical and theoretical issues that one must address. When my colleagues are faced with a new drug they frequently approach to me to ask whether they can 
'just throw fluorine on it' and use it as a tracer to tell me how the drugs behave; however, it is never that simple.

First, just 'throwing' a type of tracer on a drug may alter the metabolism or behavior of the drug or the tracer we put on may become dissociated from the drug early on, which means that you are not really imaging the distribution. Therefore, you have to really understand the biochemistry and metabolism of the agent and the tracer, as well as how they behave when given together.

Second, you have to determine how predictive the tracer is, in terms of the essential components that determine whether or not a drug is efficacious. For instance, you may have a drug that is evenly distributed in the whole body; however, if its only effect and toxicity is in the tumor, it could be a great drug but useless as an imaging agent, being completely nonpredictive. On the other hand, you may have a tracer whereby you know the drug is labeled, and goes directly towards the tumors and is taken up beautifully, but does not block the pathway; it may be an awful drug but produce beautiful images of the tumor. Therefore, it is important to marry these to demonstrate the uptake and metabolism of the tracer you are using and ensure they mirror and predict the results of the therapeutic intent, which is not an easy study to do in many cases and requires a lot of work.

\section{Q What are the main advantages of PET?}

Many drug companies are increasingly using both labeled agents as well as other metabolic agents that are not the labeled drug in order to help develop new drugs. The cost of their drug development runs into more than US\$1 billion and is very substantial; therefore, if imaging can provide any hints to drug companies of the success or failure of an agent, companies will jump on the opportunity to incorporate imaging.

Several years ago I performed a study with colleagues at a pharmaceutical company [5]. We looked at the ability of a viral agent, a modified adenovirus, to treat patients with advanced CRC. The company first approached me to conduct PET imaging every couple of months in addition to standard anatomic imaging, in order to see if there was efficacy behind what they were seeing with CT. I suggested using PET on an occasional basis to help in addition to the CT to monitor the situation in order to save money. However, it became clear that a few extra PET scans on a couple of dozen patients was nothing when compared with the costs of the next level of study, which requires building a new factory to produce the agent and to conduct trials that could involve several hundreds of patients.

Therefore, we treated approximately 18 patients, completing a CT and PET scan every couple of months, and found no real evidence of benefit with either approach, noting that the drug was clearly not behaving, as we would hope, and decided not to develop the agent any further. To the drug company this was money very well spent to help determine whether they should further pursue very costly studies in the future; therefore, I think many companies are now looking at any way they can break down their patient population and truely demonstrate efficacy.

With some patients we are increasingly seeing that targeted agents do not shrink tumors; they may change some of the consistency and growth may stop, but there is often very little shrinkage. As such, metabolic and predictive measurements obtained with PET can be very useful for both patients and for the development of the drugs, as well clinically as they are integrated into the standard of care.

\section{Q What are the limitations when conducting PET research?}

The costs of imaging are substantial; a PET scan costs thousands of US dollars, given the machinery and the tracer that needs to be synthesized for its use. On the other hand, the drugs we now routinely use cost much more, even on a monthly basis.

When I first started working in the area of PET, researchers were dubious that such an expensive and difficult imaging technique would find routine use. At that time, many of our drugs were inexpensive. The costs of PET scanning in absolute terms, and certainly relative terms, have gone

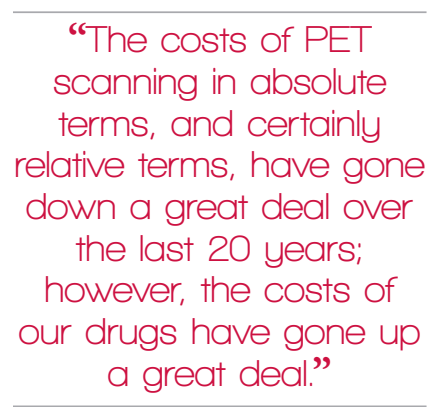




\begin{tabular}{l}
\hline "...now we have several \\
options for treating \\
patients with advanced \\
CRC; therefore, being \\
able to evaluate the \\
patients early on and \\
determine whether or not \\
a drug is working or is \\
likely to work will point \\
the way to other \\
agents."
\end{tabular}

down a great deal over the last 20 years; however, the costs of our drugs have gone up a great deal. The cost of a single PET scan has more than made up for just one dose of many drugs. Therefore, I think the interest in PET increases as we try to evaluate these agents for development and for an individual patient.

The other factor that has changed over the last 20 years is, fortunately, we now have more treatment options for many of the diseases that afflict patients with cancer. Years ago when you had one drug, which was often the situation (e.g., with CRC 20 years ago the only available drug 5 -FU), if you could tell a patient that the drug was not working then there were not a lot of other options. However, now we have several options for treating patients with advanced CRC; therefore, being able to evaluate the patients early on and determine whether or not a drug is working or is likely to work will point the way to other agents.

This is true for breast cancer, lung cancer, and now in kidney and liver cancer - there are a number of agents one can choose from; therefore, determining if an expensive agent is not really helping a patient and how we should go on and do something different, is clearly very beneficial. Fortunately, this has changed dramatically in the last couple of decades.

Q When looking at validating data, specifically for $C R C$, are there any major challenges?

I do not think that the challenges are unique to CRC; they are common problems across cancers in our study in terms of validating the results of imaging with PET and other techniques. These studies are never easy since there is often no real gold standard. The ultimate goal of our treatment is to keep people alive longer; fortunately, we have been able to do that even with people with advanced disease it used to be that the average survival of patients with advanced CRC was 6 months and now it is on the order of a couple of years.

Patients go through various rounds of treatment; therefore, if a particular agent is not working we do other things, and survival becomes a difficult end point because you do not know whether the subsequent treatment is really affecting the ultimate survival - the treatment you gave earlier may have had less of a role. Therefore, we have trouble evaluating whether one treatment or another was critical in keeping a patient alive for longer. With imaging, you know you can compare PET imaging to CT, but that is not really a gold standard. The size is certainly not the only determinant and does not tell us much about what is really going on. Therefore, these studies become more difficult because the end point gets further and further away when you are able to keep the patient alive longer and when you know the results of some of the new treatments do not particularly change tumor size - as such, sometimes you cannot use the standard anatomic imaging.

One of the routine end points we use in our studies is progression-free survival; however, to some extent, this is always an unclear measurement because you are looking at the development of new lesions, which are sometimes hard to measure. This means the comparators become more difficult, which becomes a limiting factor in the development of our imaging agents and incorporation into the studies.

\section{Q Moving forward, are there any challenges you envisage in the development of treatments for CRC?} One problem we have is the testing of new therapeutic agents on patients with CRC. As we molecularly type the disease and look for smaller and smaller subsets as we treat them, it means there are fewer patients available.

For example, at a meeting I recently attended (National Cancer Institute Colon Task Force, Chicago, IL, USA, June 2012), we were discussing trials in patients with CRC who have mutations in $B R A F$, for which there has been one recently approved agent with others also in the pipeline. The problem in developing these agents for CRC is they only represent approximately $10 \%$ of CRC patients; therefore, conducting a therapeutic and imaging trial becomes difficult as you do not have many patients, making study 
design and accrual issues very significant. As such, we are increasingly subdividing each type of disease, which makes it difficult to conduct the studies - I believe we are going to be seeing this more and more in CRC.

\section{Q Are there any suggested ways to overcome these problems?}

We are working at a national and international level to help screen patients early on for a number of molecular phenotypes in order to identify which ones will go into one trial or another. I know this is being done in the UK (FOCUS 4 trial, UK) and we have had discussions about doing it in the USA as well, where patients will come in the door and get a wide range of molecular testing done, then, as the drugs become available and studies open up, you recruit them into a particular study in order to evaluate their response against a particular target.

Therefore, people are looking at ways to overcome the issue, but it is certainly difficult to get such studies done, simply because they require a national effort, if not an international effort, to conduct them.

Q Is there a particular area of research you are interested in working in that you currently are not?

One of the areas of research we really need to get better markers on is in regards to antivascular agents. Bevacizumab is licensed and routinely used in patients with advanced CRC and, recently, at the American Society of Clinical Oncology conference (IL, USA), a study showed that even after patients have progressed in their first-line chemotherapy, the continued use of bevacizumab provides some benefit [6] . However, those benefits, even in the firstand second-line settings, are still relatively small - only $10 \%$ of the patients may benefit while we are treating 100 patients who have advanced disease and are receiving a very expensive drug with its share of toxicity.

Researchers have been looking for molecular markers to predict which patients would really benefit from a treatment such as bevacizumab, and they look at markers based on the tumor. However, people have now also started to look at various imaging approaches, considering whether they can demonstrate if the bevacizumab is really having an effect, which would provide a way to determine which patients we should recieve this agent - and if a response can be seen with PET.

There are a number of ways that scientists are looking at vascularity with PET and tracers that bind to the vascular space, as well as with MR, but so far we have not really found anything that is predictive. I feel further work in this area is essential in order to really understand who is going to benefit from such treatments. We have a number of vascular agents that are in the pipeline and approved, with new ones becoming available. For example, there is a new second-line agent that I think will probably be approved in the USA in the next few months that is an antivascular agent (aflibercept); however, we do not have any good markers to determine which patients are benefiting the most from it.

\section{Q Do you think that work on} antivascular agents will be a major part of your work in the future?

I have looked into it and had discussions with some of the companies developing such agents. The biggest problem I have had is trying to figure out how to do such a trial, and how to make it simple and designed to get reasonable amounts of data. Bevacizumab by itself, for instance, has little activity in patients with advanced CRC and is routinely given with chemotherapy. There are some data demonstrating that it may work in a maintenance setting, and in fact, a new trial combining erlotinib also looks promising, but it is not used in the front-line treatment of the disease.

Therefore, I have had discussions with some of the companies developing imaging agents for vascularity on how to best study it. However, it is difficult to do such studies, to plan them and gauge what will be very useful. More work is ongoing, and I am sure I will have further discussions in order to try and design such a trial; however, at this point, I must say we do not have an open trial to address this question.

\footnotetext{
"There are a number of ways that scientists are looking at vascularity with

PET and tracers that bind to the vascular space, as well as with MR, but so far we have not really found anything that is predictive."
} 


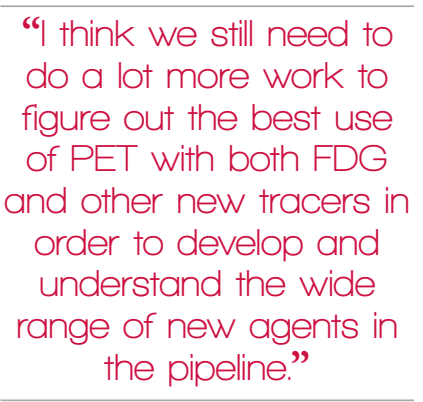

"I think we still need to do a lot more work to figure out the best use of PET with both FDG and other new tracers in order to develop and understand the wide the pipeline."
Q What future research do you feel is most important for improving the quality of PET for CRC?

Getting new markers and new approaches in order to help us evaluate targeted agents and approaches is critical and sometimes requires a number of different tracers depending on the disease, target and therapy. Simply knowing the target is there based on a molecular genotype does not tell you that the target necessarily drives the tumor; however, it is certainly the first step, and imaging can help us there.

It is important to know if the target is certainly active, and if altering it will change the metabolism that ultimately responds to the tumor. Therefore, you are looking for a series of markers that will give you different amounts of information about the tumor and its response to therapy.

Q Is there an example where this has been effectively done?

I think it has probably been best done in breast cancer; for instance, knowing a patient has an estrogen receptor in their tumor will help predict whether antiestrogen therapy will potentially be useful. In general, not having the estrogen receptor has been found to be predictive that such treatments are not efficacious. On the other hand, in those with the receptor antiestrogen therapy given alone, it is useful approximately $20 \%$ of the time, so clearly that is the first step, but not the last step.

Research has been looking at labeling estrogens and is showing that even when you take out the tumor and stain it, the receptor may be there; it does not take up a labeled estrogen as imaged with PET, so simply having the receptor there does not inform you whether or not it is an active receptor or available for binding [7]. As such, that is the second step, and PET is useful in this setting - the response rate may be $40 \%$ when using PET to help select your patients. In this sense, you have done better than just looking at the genomics or looking at the proteins in the tumor, but you still have not made it all the way there.
Consequently, you then have to look at the actual pathway and see whether or not it is active and if it can be modified. People have been given small doses of estrogen for 1 day and shown that the tumor is affected and is found to be almost completely predictive of a response.

Q Is such work applicable to CRC?

Yes, in light of research conducted in breast cancer there are certain things we have to be aware of in CRC: do we have a measure that could be a molecular measure (i.e., an altered gene or a receptor present); is it really an active receptor or pathway and is it a driver receptor or pathway; finally, does the agent we have that attaches to or affects that pathway really alter the ultimate growth or metabolism of the tumor? As such, you may end up looking at a number of steps, which I believe we should do with CRC, in order to determine how to do it with each of the new agents available.

Q Is this the sort of research that you are currently working on at the Karmanos Cancer Institute?

Yes, we are looking at different ways to measure the response to treatment and particular pathways [101]. For instance, the oldest drug we use in CRC, which has been around since in the 1950s, is 5-FU. Previously, people labeled 5-FU for PET and there were some data showing that the uptake of 5-FU was potentially predictive. Patients who only took up a little drug did not benefit much. However, this has not really been utilized to any great extent.

We have been looking at using FLT in some of our studies because it is taken up and altered in the same pathway that 5-FU uses. There has been work by others demonstrating a 'flare phenomena', in that after 5-FU is administered there is increased uptake of FLT within hours to a day. This is because you block the thymidine synthesis pathway and the cell tries to compensate by taking more FLT; therefore, this flare may help predict the patients who are benefiting from the 5-FU.

As such, we have been looking at this and are planning further studies to determine whether the agent is doing the job we think it should. 
Q In summary, how you feel the field of PET in CRC is advancing?

At this point in time, PET with FDG is certainly used routinely in the treatment of CRC; however, I think we still need to do a lot more work to figure out the best use of PET with both FDG and other new tracers in order to develop and understand the wide range of new agents in the pipeline. We still do not have a good handle on how to predict and monitor the targeted agents we have.

Recently at the American Society of Clinical Oncology conference, work has been presented on regorafenib, showing benefits in the third-line therapy of patients with advanced CRC [8]. However, we do not have a predictive marker available or a way to monitor the patients very well. The increase in survival was on the order of a month and a half for such patients; therefore, we clearly need to figure out which patients are likely to benefit and are benefiting from this new drug. I must say, the biggest frustration is we do not have a way to do that yet, whether with a molecular marker or an imaging marker - as such, I would say at this point, current research needs a new push to determine how to best incorporate such techniques.

\section{Financial \& competing interests}

\section{disclosure}

A Shields has received funding from $\mathrm{NIH}$. He has no other relevant affiliations or financial involvement with any organization or entity with a financial interest in or financial conflict with the subject matter or materials discussed in the manuscript apart from those disclosed.

No writing assistance was utilized in the production of this manuscript.

\section{References}

1 Ruers TJ, Wiering B, van der Sijp JR et al. Improved selection of patients for hepatic surgery of colorectal liver metastases with ${ }^{18}$ F-FDG PET: a randomized study. J. Nucl. Med. 50(7), 1036-1041 (2009).

2 Wieder HA, Geinitz H, Rosenberg R et al. PET imaging with $\left[{ }^{18} \mathrm{~F}\right] 3^{\prime}$-deoxy-3'fluorothymidine for prediction of response to neoadjuvant treatment in patients with rectal cancer. Eur. J. Nucl. Med. Mol. Imaging 34(6), 878-883 (2007).

3 Dehdashti F, Grigsby PW, Myerson RJ, Nalbantoglu I, Ma C, Siegel BA. Positron emission tomography with $\left[{ }^{18} \mathrm{~F}\right]-3^{\prime}$-deoxy3'fluorothymidine (FLT) as a predictor of outcome in patients with locally advanced resectable rectal cancer: a pilot study.
Mol. Imaging Biol. doi:10.1007/s11307-0120566-y (2012) (Epub ahead of print).

4 Sun H, Collins JM, Mangner TJ, Muzik O, Shields AF. Imaging the pharmacokinetics of [F-18]FAU in patients with tumors: PET studies. Cancer Chemother. Pharmacol. 57, 343-348 (2006).

5 Hamid O, Varterasian ML, Wadler S et al. Phase II trial of intravenous CI-1042 in patients with metastatic colorectal cancer. J. Clin. Oncol. 21, 1498-1504 (2003).

6 Arnold D, Andre T, Bennouna J et al. Bevacizumab (BEV) plus chemotherapy (CT) continued beyond first progression in patients with metastatic colorectal cancer (mCRC) previously treated with BEV plus CT: results of a randomized Phase III intergroup study (TML study). J. Clin.
Oncol. 30 (Suppl.) Abstract CRA3503 (2012).

7 Linden HM, Stekhova SA, Link JM et al. Quantitative fluoroestradiol positron emission tomography imaging predicts response to endocrine treatment in breast cancer. J. Clin. Oncol. 24(18), 2793-2799 (2006).

8 Van Cutsem E, Sobrero AF, Siena F et al. Phase III CORRECT trial of regorafenib in metastatic colorectal cancer (mCRC). J. Clin. Oncol. 30 (Suppl.) (Abstract 3502) (2012).

\section{- Website}

101 Safety and Efficacy Study of FLT Radiolabeled Imaging Agent With PET Scan in Invasive Breast Cancer. http://clinicaltrials.gov/ct2/show/ NCT00572728 